\title{
The Treaty of Influenza and SARS-CoV-2: Titans Versus Olympians
}

\author{
Ilad Alavi Darazam (iD) ${ }^{1,2,{ }^{*}}$, Masoud Mardani (iD ${ }^{1,2}$, Mohammad Mahdi Rabiei (iD) ${ }^{1,2}$, Firouze Hatami ${ }^{1,2}$ \\ and Farid Javandoust Gharehbagh ${ }^{1,2}$ \\ ${ }^{1}$ Department of Infectious Diseases, Loghman Hakim Hospital, Shahid Beheshti University of Medical Sciences, Tehran, Iran \\ ${ }^{2}$ Infectious Diseases and Tropical Medicine Research Center, Shahid Beheshti University of Medical Sciences, Tehran, Iran \\ "Corresponding author: Department of Infectious Diseases, Loghman Hakim Hospital, Shahid Beheshti University of Medical Sciences, Tehran, Iran. Email: ilad13@yahoo.com
}

Received 2020 December 19; Revised 2021 January 02; Accepted 2021 January 02.

Keywords: Influenza, Epidemic, Pandemic, COVID-19, SARS-CoV-2

A major concern for healthcare systems is the occurrence of the annual influenza epidemics. With the arrival of winter in almost all regions with different climates, seasonal influenza is more probable to occur. Depending on the distance from the equator, the occurrence of seasonal influenza can be varied (1). Influenza is responsible for 3 5 million severe infections. The annual mortality rate for influenza has been reported to be 290,000 - 650,000 (2).

With the commencement of flu season in the northern hemisphere, the high prevalence of COVID-19 may become more dangerous relative to other seasons. The simultaneous outbreak of influenza and COVID-19 poses many adverse effects on the healthcare system. Concerning overlaps between flu and COVID-19 symptoms, the lack of laboratory tests with promising sensitivity and specificity, as well as precise diagnostic image findings, has raised concerns over the proper distinguishing of influenza and COVID-19.

The sensitivity and specificity of influenza tests depend on testing methods, time of specimen collection, and source and quality of the specimen. Rapid Influenza Diagnostic tests (RIDTs) can detect the influenza antigen in 10 15 minutes, but they offer only $50 \%-70 \%$ sensitivity and are not commercially available (3).

As known, RT-PCR from nasopharyngeal swabs is the gold standard with $60 \%-70 \%$ sensitivity (but lower in realworld) as a diagnostic test for SARS-CoV-2 (4). Molecular tests such as RT-PCR and PCR are not reliable. Molecular tests for influenza and COVID-19 can also produce falsepositive or false-negative results. Each molecular result should be interpreted individually for each case according to clinical manifestations since the tests are not entirely able to rule in or out either influenza or COVID-19 cases. The fact is of note, that timely diagnosis of infection is pivotal for clinicians to implement appropriate clinical management. As mentioned above, there are currently no reliable laboratory tests with proper efficacy for the diagnosis of influenza and COVID-19.

Today, although intensive efforts are being made globally to identify an effective treatment, there is still no proven treatment for COVID-19 and its complications. The absence of exact pre-defined criteria to discern influenza from COVID-19 leads to the misclassification of patients. Co-infection is expected in hospitalized patients with either COVID-19 or influenza who have been incorrectly hospitalized in the wrong wards. Therefore, the mortality rate and the incidence of respiratory failure will increase.

Sticking to instructions for the prevention of these overwhelming contagious diseases is a key factor to cease terrible outcomes. Both standard precautions and transmission-based precautions are used in medical centers and hospitals. Prevention actions in society, such as personal protective strategies (i.e., hand hygiene, selfquarantine, and face mask) and mitigate strategies (i.e., social distance, prolonged school closure), can be useful for the problem (5). However, it is a great dilemma that the lack of acceptance, compliance, and adherence to these principles is dominantly seen.

Another principle of prevention is an effective and widespread implementation of vaccination. Although different COVID-19 vaccines are under development, there is currently no effective and safe vaccine. The COVID-19 vaccination trials have not been performed in a large sample size and have not been evaluated in a long period. Besides, the prevention potency of antibodies against the COVID-19 virus is unknown. 
Previous studies demonstrated that immunization by vaccination provides effective protection against influenza (6). The effectiveness rate of influenza vaccines has been estimated to be $25 \%$ - $60 \%$ annually (7). Influenza viruses constantly change through a process called antigenic drift; therefore, new vaccines should be produced each year. Based on resource documents provided by the WHO, influenza vaccination offers social benefits and is costeffective (8). Vaccination can reduce the number of patients, hospitalization, mortality rate, and respiratory failure. Usually, the flu vaccine is prescribed once a year at the opening days of flu season. Due to the scarcity of vaccines, only high-risk people who are susceptible to serious complications are vaccinated. However, regarding the fact that the main aim of vaccination is to stop the spreading of the disease and its transmission, the high-risk people vaccination policy has not been satisfying. Widespread vaccination, therefore, is a priority, at least for those who are in contact with high-risk people.

Like influenza, COVID-19 has the potential to become a seasonal disease (9). The high infectivity and the long incubation period, along with many patients who experience mild-to-moderate symptoms, can make COVID-19 a troubling disease. A vaccine is crucial because data show the asymptomatic transmission of COVID-19 (10-12). The ability of coronaviruses to make a pandemic was predicted more than 10 years ago by scientists (13). Despite the warnings, the world was not able to manage this pandemic. The distribution of a trustworthy vaccine to the global population might be the most effective approach to end this pandemic and might be the only way to quell restrictions. Challenges included the vaccine design, its manufacture, and global distribution. Cold chain requirements present logistical and fiscal barriers to the availability of important, life-saving vaccines in resource-poor areas of the world. Introducing new vaccine delivery platforms and devices to break cold chain limitations can be efficient solutions to safeguard potent vaccination for both wealthy and lowerincome countries.

Pfizer and BioNTech's BNT162b2, which was the first vaccine accepted by the US Food and Drug Administration (FDA) for emergency use authorization, the mRNA-1273 vaccine developed by the US biotech company Moderna in partnership with the US National Institutes of Health (NIH), the Sinopharm vaccine by China, the University of Oxford and AstraZeneca vaccine, and the Sputnik V vaccine by Russia are promising to prevent COVID-19.

The end of 2020 and winter of 2021 will be more challenging times due to the probable co-circulation of both in- fluenza and SARS-CoV-2 in the northern hemisphere. Thus, influenza vaccine demand will increase all over the world. There should be an appropriate management plan for providing and distributing influenza vaccines to facilitate their availability. Wrong policies can lead to profiteering, swindle, and people accumulation who are demanding for vaccines. The latter paves the way for the increased number of infected people. Hence, to minimize the number of people not willing to be vaccinated by making various alibis, providing easy access to the vaccine should be a concern, and proper decisions should be made.

\section{Footnotes}

Authors' Contribution: All authors contributed equally. Conflict of Interests: Not applicable.

Funding/Support: Not applicable.

\section{References}

1. Li Y, Reeves RM, Wang X, Bassat Q, Brooks WA, Cohen C, et al. Global patterns in monthly activity of influenza virus, respiratory syncytial virus, parainfluenza virus, and metapneumovirus: a systematic analysis. Lancet Glob Health. 2019;7(8):e1031-45. doi: 10.1016/S2214109X(19)30264-5. [PubMed: 31303294].

2. World Health Organization. Influenza (seasonal). 2018. Available from: https://www.who.int/news-room/fact-sheets/detail/influenza(seasonal).

3. Centers for Disease Control and Prevention (CDC). Overview of In fluenza Testing Methods. 2020. Available from: https://www.cdc.gov/ flu/professionals/diagnosis/overview-testing-methods.htm.

4. Duarte ML, Santos LRD, Contencas ACS, Iared W, Peccin MS, Atallah AN Reverse-transcriptase polymerase chain reaction versus chest computed tomography for detecting early symptoms of COVID-19. A diagnostic accuracy systematic review and meta-analysis. Sao Paulo Med J. 2020;138(5):422-32. doi: 10.1590/1516-3180.2020.034306072020. [PubMed: 32844901]

5. Qualls N, Levitt A, Kanade N, Wright-Jegede N, Dopson S, Biggerstaff M, et al. Community Mitigation Guidelines to Prevent Pandemic Influenza - United States, 2017. MMWR Recomm Rep. 2017;66(1):1-34. doi: 10.15585/mmwr.rr6601a1. [PubMed: 28426646]. [PubMed Central: PMC5837128]

6. Alavi Darazam I, Mardani M. A Trilogy of Influenza, Immunization, and Antimicrobial Resistance: A Review Article. Arch Clin Infect Dis 2019;14(2).

7. Centers for Disease Control and Prevention. CDC seasonal flu vaccine effectiveness studies. 2020. Available from: https://www.cdc.gov/flu/ vaccines-work/effectiveness-studies.htm.

8. World Health Organization. Influenza vaccines. 2020. Available from: https://www.who.int/immunization/research/development/ influenza/en/index1.html.

9. Kissler SM, Tedijanto C, Goldstein E, Grad YH, Lipsitch M. Projecting the transmission dynamics of SARS-CoV-2 through the postpandemic period. Science. 2020;368(6493):860-8. doi: 10.1126/science.abb5793. [PubMed: 32291278]. [PubMed Central: PMC7164482]. 
10. Day M. Covid-19: four fifths of cases are asymptomatic, China figures indicate. BMJ. 2020;369:m1375. doi: 10.1136/bmj.m1375. [PubMed: 32241884].

11. Sutton D, Fuchs K, D'Alton M, Goffman D. Universal Screening for SARS-CoV-2 in Women Admitted for Delivery. $N$ Engl $J$ Med. 2020;382(22):2163-4. doi: 10.1056/NEJMc2009316. [PubMed: 32283004]. [PubMed Central: PMC7175422].

12. Mizumoto K, Kagaya K, Zarebski A, Chowell G. Estimating the asymp- tomatic proportion of coronavirus disease 2019 (COVID-19) cases on board the Diamond Princess cruise ship, Yokohama, Japan, 2020. Euro Surveill. 2020;25(10). doi: 10.2807/1560-7917.ES.2020.25.10.2000180. [PubMed: 32183930]. [PubMed Central: PMC7078829].

13. Cheng VC, Lau SK, Woo PC, Yuen KY. Severe acute respiratory syndrome coronavirus as an agent of emerging and reemerging infection. Clin Microbiol Rev. 2007;20(4):660-94. doi: 10.1128/CMR.0002307. [PubMed: 17934078]. [PubMed Central: PMC2176051]. 